\title{
Medicina Baseada em Evidências: Instrumento para Educação Médica Permanente entre Psiquiatras?
}

\author{
Evidence-Based Medicine: a Tool for Continuing \\ Medical Education Among Psychiatrists?
}

\author{
Isabela Yuri Tsuji \\ Liliana Cavalcanti Zamperin ${ }^{I}$ \\ Selma Rumiko Tsuji ${ }^{I}$ \\ Valéria Garcia Caputo ${ }^{I}$
}

\section{PALAVRAS-CHAVE: \\ - Educação Médica. \\ - Psiquiatria. \\ - Epidemiologia Descritiva.}

\section{KEY-WORDS}

- Medical Education.

- Psychiatry.

- Epidemiology, Descriptive

Recebido em: 19/11/2008

Reencaminhado em: 03/04/2009

Aprovado em: 22/08/2009

REVISTA BRASILEIRA DE EDUCAÇÃO MÉDICA

\section{RESUMO}

Introdução: $O$ médico atualizado pode oferecer o melhor cuidado ao paciente e evitar consequências negativas que a defasagem científica pode acarretar. Objetivos: Identificar os meios de atualização utilizados pelos psiquiatras brasileiros; avaliar seus conhecimentos sobre Medicina Baseada em Evidências (MBE) e sua utilização na educação permanente. Método: Estudo transversal realizado no XXIV Congresso Brasileiro de Psiquiatria. Os participantes $(n=188)$ responderam um questionário anônimo autoaplicado, com 28 perguntas sobre características sociodemográficas, fontes e periodicidade de atualização, e conhecimentos sobre MBE. Resultados: Para atualização de conhecimentos, 98,3\% utilizavam os congressos brasileiros; $97,9 \%$ as revistas nacionais; $93,9 \%$ os livros-texto; $89,9 \%$ as revistas das indústrias farmacêuticas; $63,5 \%$ os consensos brasileiros; $63,3 \%$ a base de dados Medline; $56,7 \%$ as revistas internacionais; e 35\% a Biblioteca Cochrane. Os fatores estatisticamente significativos associados com bom conhecimento sobre MBE foram estar graduados há menos de dez anos ( $p<0,001)$, usar o Medline $(p<0,009)$ e a Biblioteca Cochrane $(p<0,03)$ como fonte de busca de literatura médica. Conclusões: Os psiquiatras fazem pouco uso da melhor fonte de evidência para educação médica permanente e continuada, havendo, assim, menor beneficio aos pacientes na tomada de decisão clínica.

\footnotetext{
Background: Up-to-date psychiatrists provide better patient care and avoid the potentially negative consequences of outdated scientific knowledge. Objectives: The aim was to identify how Brazilian psychiatrists update their skills, and to evaluate their knowledge of evidenced-based medicine (EBM) and its use in continuing medical education. Method: This was a cross-sectional study conducted at the $24^{\text {th }}$ Brazilian Congress of Psychiatry. Participants $(n=188)$ answered an anonymous, selfapplied questionnaire with 28 questions on socio-demographic characteristics, sources and frequency of updating, and knowledge of EBM. Results: To update their knowledge, 98.3\% of the sample attended Brazilian congresses, 97.9\% read Brazilian medical journals, 93.9\% used textbooks, 89.9\% consulted journals published by pharmaceutical companies, $63.5 \%$ accessed the Brazilian guidelines, $63.3 \%$ used the Medline database, 56.7\% read international journals, and 35\% accessed the Cochrane Library. Factors that showed statistically significant association with good knowledge of EBM were having graduated from medical school less than ten years before $(p<0.001)$ and use of Medline $(p<$ $0.009)$ and Cochrane Library $(p<0.03)$ as sources for searching the medical literature. Conclusions: Brazilian psychiatrists make limited use of the best sources of evidence for continuing medical education, thus producing less benefit for patients in clinical decision-making.
} 


\section{INTRODUÇÃO}

Na tentativa de estabelecer uma relação empática e aliviar o sofrimento do paciente, devem-se considerar suas expectativas e preferências, sem, no entanto, esquecer o direito dos indivíduos de receberem o que de melhor a ciência pode oferecer em termos de cuidado à saúde. O Código de Ética Médica (CEM), em alguns de seus artigos, trata desse compromisso que o médico deve ter de estar sempre atualizado e capacitado para oferecer a melhor atenção ao seu paciente ${ }^{1}$.

A atualização dos conhecimentos é um processo dinâmico que envolve três etapas: o reconhecimento da necessidade de informações, a obtenção das mesmas e a determinação do seu valor científico. Quanto melhor for um estudo do ponto de vista metodológico, mais confiáveis e generalizáveis serão as conclusões que se podem tirar, aumentando seu valor científico ${ }^{2}$.

Nesse contexto, insere-se a Medicina Baseada em Evidências (MBE), termo criado e utilizado por um grupo de pesquisadores da Faculdade de Medicina da Universidade de McMaster, no Canadá na década de 1980, para denominar uma estratégia de aprendizagem que envolve as seguintes etapas: (a) realizar questões clínicas bem formuladas; (b) localizar as informações disponíveis na literatura; (c) avaliar criticamente as informações relevantes; (d) utilizar criticamente as informações avaliadas, para a tomada de decisão clínica ${ }^{3}$.

Esse novo paradigma reconhece a importância da experiência clínica, e mesmo da intuição diagnóstica, mas sublinha que o registro sistemático das observações de maneira reprodutível e não preconceituosa pode aumentar a certeza do diagnóstico, a eficácia da terapêutica e a confiança no prognóstico ${ }^{4}$.

Para a boa prática da BEM, é necessário que médicos pesquisadores entendam as particularidades de um protocolo. Contudo, os médicos "não pesquisadores", aqueles que buscam a informação, devem compreender os princípios básicos da pesquisa com o objetivo de interpretar a validade dos resultados do estudo publicado ou apresentado 5 .

Assim, uma das estratégias para equacionar a necessidade do médico de se manter bem informado com o pouco tempo de que dispõe seria aprender a selecionar os artigos que realmente merecem ser $\operatorname{lidos}^{6}$. Na prática, mais de 2 mil artigos são publicados diariamente no Medline, gerando mudanças rápidas na área da saúde. E estas informações nem sempre chegam aos profissionais em tempo e forma adequados ${ }^{7}$.

Tendo em vista a relevância da atualização científica na medicina e as repercussões negativas que a defasagem no conhecimento médico individual pode acarretar tanto para o pa- ciente como para o médico, é necessário avaliar a postura do profissional médico frente à sua aprendizagem permanente $\mathrm{e}$ embasamento científico. Desta forma, este estudo teve o objetivo de identificar os meios e a periodicidade de atualização dos médicos psiquiatras, bem como avaliar seus conhecimentos acerca dos princípios da MBE e verificar se estes têm sido utilizados como instrumento de educação permanente.

\section{MÉTODO}

Foi realizado um estudo epidemiológico observacional, descritivo e transversal, com uma amostra de conveniência de médicos psiquiatras abordados aleatoriamente nas dependências do XXIV CBP, realizado em Curitiba (PR) de 24 a 28 de outubro de 2006. Uma vez explicado o objetivo da pesquisa, o médico que aceitasse participar assinava o Termo de Consentimento Informado.

O protocolo de pesquisa foi previamente submetido e aprovado pelo Comitê de Ética em Pesquisa Envolvendo Seres Humanos da Faculdade de Medicina de Marília — Famema (CEP n⿳0 110/06).

O instrumento para a coleta dos dados foi um questionário autoaplicado com 28 questões, sendo 8 relacionadas às características sociodemográficas da população de estudo (gênero, idade, local de trabalho, ano de graduação, titulação, número de atendimentos diários); 12 questões de associação simples quanto a fontes e periodicidade de atualização; e 8 questões de múltipla escolha de conhecimentos sobre MBE, nas quais apenas uma resposta é correta e três são erradas. As oito questões sobre MBE abordavam as características de um estudo ideal para avaliar evidência terapêutica, prevalência de patologia, associação correta entre intervenção clínica e tipo de estudo, interpretação de resultados de estudo de acurácia diagnóstica, avaliação do nível de evidência científica para confiabilidade e precisão, e interpretação de um gráfico de metanálise. O conteúdo abordado foi retirado do livro Evidence-Based Medicine - How to Practice E Teach EBM ${ }^{8}$.

$\mathrm{Na}$ análise, os dados dicotômicos foram apresentados como frequência e percentagem. Foi realizada análise de regressão logística para avaliação de possível associação significativa de algum fator. Foi considerado estatisticamente significante se $p<0,05$.

\section{RESULTADOS}

Participaram do estudo 188 psiquiatras, o que representou $3,65 \%$ de todos os inscritos (5.138) no congresso. Metade dos congressistas tinha entre 11 e 30 anos de formação, e a maioria $(63,3 \%)$ possuía título de especialista. As principais características da amostra estudada estão descritas na Tabela 1. 
Tabela 1

Características dos psiquiatras $(\mathrm{n}=188)$ do XXIV Congresso Brasileiro de Psiquiatra, Curitiba (PR), 2006

\begin{tabular}{|c|c|c|}
\hline Característica & $\mathrm{N}$ & $(\%)$ \\
\hline \multicolumn{3}{|l|}{ Idade } \\
\hline 25 a 45 anos & 87 & 46,3 \\
\hline 46 a 65 anos & 96 & 49,5 \\
\hline$>65$ anos & 8 & 4,3 \\
\hline \multicolumn{3}{|l|}{ Anos de formação } \\
\hline Até 10 anos & 61 & 32,5 \\
\hline 11 a 20 anos & 36 & 19,1 \\
\hline 21 a 30 anos & 58 & 30,9 \\
\hline$>30$ anos & 33 & 17,6 \\
\hline \multicolumn{3}{|l|}{ Título de especialista } \\
\hline Sim & 119 & 63,3 \\
\hline Não & 65 & 34,6 \\
\hline Não informaram & 04 & 2,1 \\
\hline \multicolumn{3}{|l|}{ Pós-graduação } \\
\hline Não tem & 69 & 36,7 \\
\hline Especialização & 87 & 46,3 \\
\hline Mestrado & 16 & 8,5 \\
\hline Doutorado & 7 & 3,7 \\
\hline Livre docência & 3 & 1,6 \\
\hline Não informaram & 6 & 3,2 \\
\hline \multicolumn{3}{|l|}{ Professor universitário } \\
\hline Sim & 33 & 17,6 \\
\hline Não & 143 & 76,1 \\
\hline Não informaram & 12 & 6,4 \\
\hline
\end{tabular}

$\mathrm{N}$ - número absoluto.
As maiores dificuldades apontadas pelos psiquiatras para atualização médica permanente foram o pouco tempo disponível (80\% dos participantes), dificuldades financeiras $(22,2 \%)$, dificuldades com o idioma inglês $(19,5 \%)$ e dificuldades no uso de recursos da internet (6,5\%) (Gráfico 1-A).

Quanto às formas de atualização médica, observou-se que 98,3\% dos participantes têm como principal fonte os congressos brasileiros, seguidos das revistas nacionais $(97,9 \%)$, revistas internacionais $(56,7 \%)$, jornais $(89,9 \%)$, indústria farmacêutica $(88,9 \%)$, consenso brasileiro (63,5\%), Medline (63,3\%), Biblioteca Cochrane (35\%) e livros-texto (93,9\%) (Gráfico 1-B).

Quanto ao desempenho dos psiquiatras relacionado ao conhecimento em Medicina Baseada em Evidências (MBE), a Tabela 2 mostra as frequências de erros, acertos e falta de resposta. A percentagem de questões não respondidas variou de acordo com cada pergunta, sendo alta naquela que se referiu à interpretação dos resultados de uma metanálise.

$\mathrm{Na}$ análise de algumas características da amostra que, em hipótese, poderiam influenciar esta frequência de acertos e erros, observou-se que os psiquiatras com tempo de formação de até dez anos ( $p<0,001)$ (Gráfico 2) e os que utilizavam o banco de dados Medline apresentaram associação estatisticamente significante nas questões sobre evidências para decisão terapêutica $(82 / 188-71,3 \%, p<0,003)$, característica do estudo de prevalência $(57 / 188-49,6 \%$, p < 0,002), critério de seleção de um artigo para avaliar tratamento (61/188 - 53\%, $\mathrm{p}<0,001)$, associações entre a pergunta clínica e o tipo de estudo $(54 / 188-47 \%$, p < 0,002), e interpretação de testes diagnósticos (77/188 - 67,5\%, p 0,009). Aqueles que utilizavam a Biblioteca Cochrane como fonte de atualização apresentaram acertos com significância estatística nas questões sobre critério

Tabela 2

Relação das questões aplicadas e sua percentagem de acertos nos psiquiatras do XXIV Congresso Brasileiro de Psiquiatria

\begin{tabular}{|c|c|c|c|c|c|c|}
\hline \multirow{2}{*}{ Tema da questão } & \multicolumn{2}{|c|}{ Acertos } & \multicolumn{2}{|c|}{ Erros } & \multicolumn{2}{|c|}{ Em branco } \\
\hline & $\mathrm{N}$ & $\%$ & $\mathrm{~N}$ & $\%$ & $\mathrm{~N}$ & $\%$ \\
\hline Qual o estudo ideal para a decisão terapêutica & 118 & 62,8 & 59 & 31,4 & 11 & 5,9 \\
\hline Qual o estudo ideal para saber uma prevalência & 76 & 40,4 & 98 & 52,1 & 14 & 7,4 \\
\hline Critério para seleção de artigo na decisão terapêutica & 81 & 43,1 & 89 & 47,3 & 18 & 9,6 \\
\hline Associação: pergunta clínica/tipo de estudo adequado & 70 & 37,2 & 93 & 49,5 & 25 & 13,3 \\
\hline Conceito de teste falso-positivo & 158 & 84,0 & 12 & 6,4 & 18 & 9,6 \\
\hline Conceito de sensibilidade de teste diagnóstico & 111 & 59,0 & 58 & 30,9 & 19 & 10,1 \\
\hline Níveis de evidência & 114 & 60,6 & 51 & 27,1 & 23 & 12,2 \\
\hline Interpretação de gráfico de metanálise & 38 & 20,2 & 95 & 50,5 & 55 & 29,3 \\
\hline
\end{tabular}




\section{Gráfico 1}

Dificuldades encontradas para atualização médica (A) e fontes mais usadas (B) entre os médicos participantes do XXIV

Congresso Brasileiro de Psiquiatria $(n=188)$.
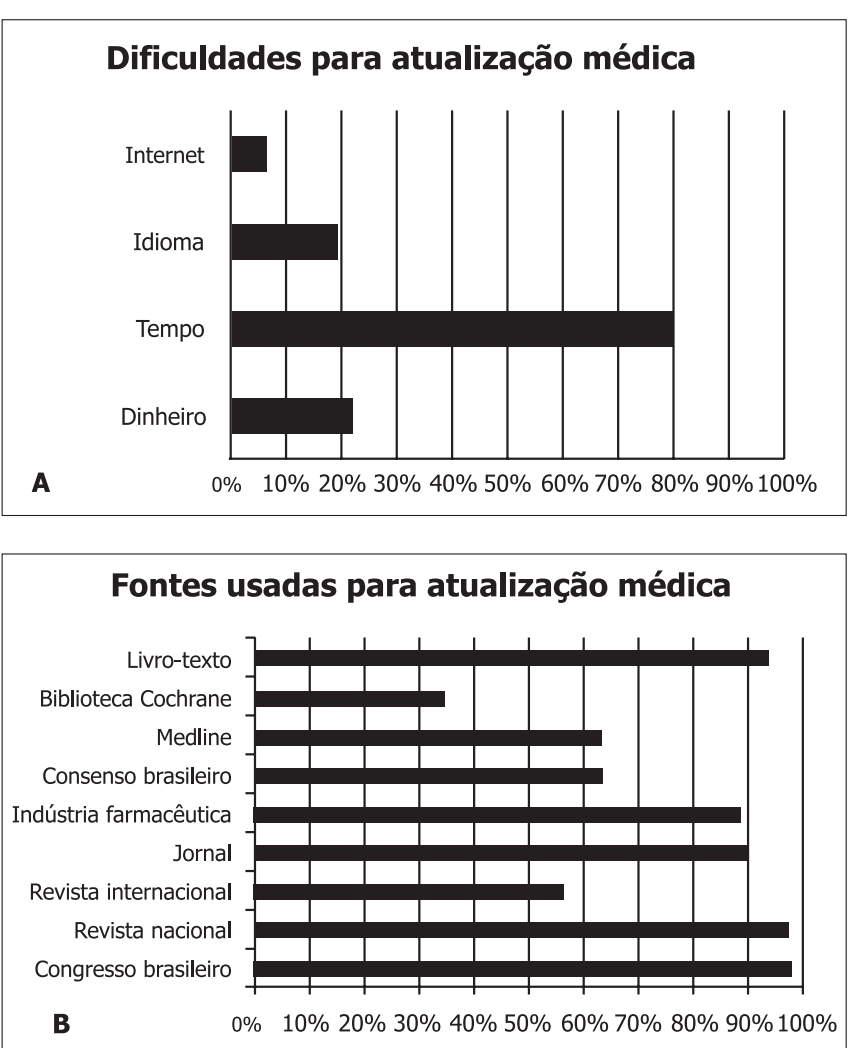

Gráfico 2

Percentagem de acerto nas questões teóricas para adequada utilização da Medicina Baseada em Evidências (MBE) e relacionamento com anos de formação médica

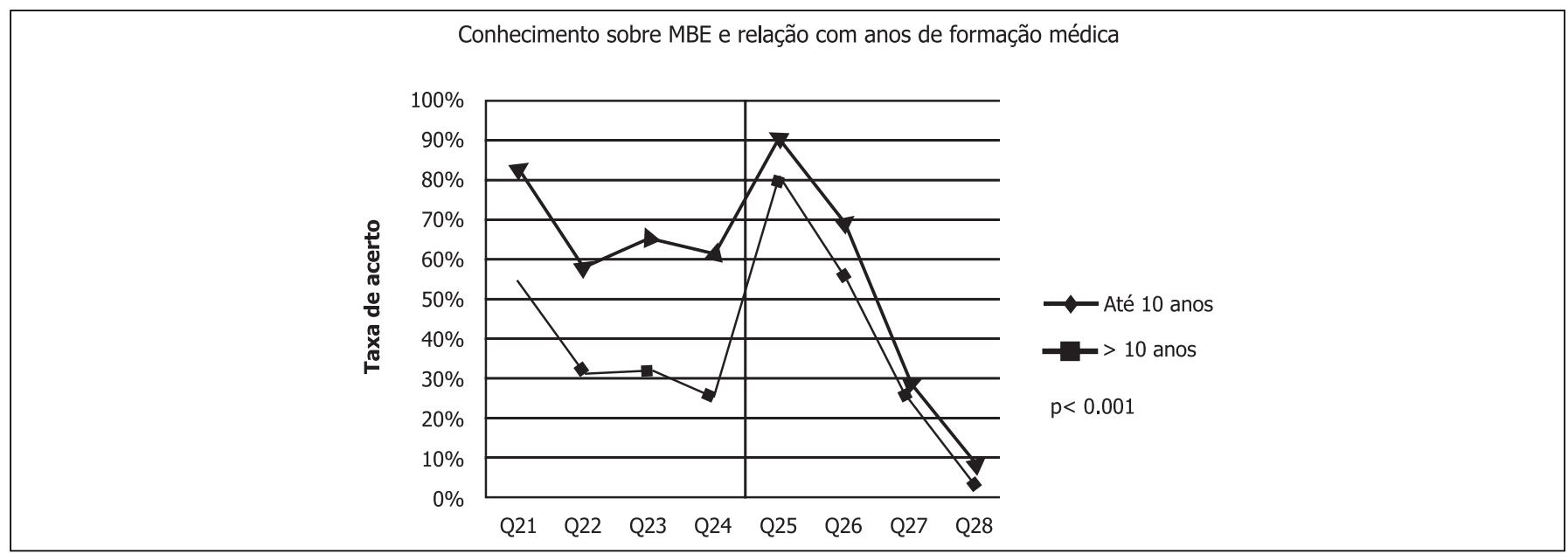

Nota: (Q21 — Qual o estudo ideal para a decisão terapêutica; Q22 — Qual o estudo ideal para saber prevalência; Q23 — Critério para seleção de artigo na decisão terapêutica; Q24 - Associação: pergunta clínica/tipo de estudo adequado; Q25 - Conceito de teste falso-positivo; Q26 - Conceito de sensibilidade de teste diagnóstico; Q27 — Níveis de evidência; Q28 — Interpretação de gráfico de metanálise). 
Tabela 3

Associação entre o número de acertos em cada questão e o tempo de formação, meio de atualização e atividade docente dos 188 psiquiatras. XXIV CBP — Curitiba, PR, Brasil, 2006.

\begin{tabular}{|c|c|c|c|c|c|c|c|c|}
\hline Questões & $\begin{array}{l}\text { Tipo de estudo } \\
\text { para tratamento }\end{array}$ & $\mathrm{p}^{*}$ & $\begin{array}{l}\text { Tipo de } \\
\text { estudo para } \\
\text { prevalência }\end{array}$ & $\mathrm{p}^{*}$ & $\begin{array}{c}\text { Como } \\
\text { selecionar } \\
\text { artigo }\end{array}$ & $\mathrm{p}^{*}$ & $\begin{array}{l}\text { Associação } \\
\text { pergunta clínica/ } \\
\text { tipo de estudo }\end{array}$ & $\mathrm{p}^{*}$ \\
\hline Variáveis & $\%$ & & $\%$ & & $\%$ & & $\%$ & \\
\hline \multicolumn{9}{|l|}{ Tempo de formação } \\
\hline Até 10 anos & 82,0 & $<0,001$ & 59,0 & $<0,001$ & 65,6 & $<0,001$ & 62,3 & $<0,001$ \\
\hline$<10$ anos & 53,5 & & 31,5 & & 32,3 & & 25,2 & \\
\hline \multicolumn{9}{|l|}{ Meio de atualização } \\
\hline Cochrane & 72,6 & 0,76 & 48,4 & 0,19 & 59,7 & 0,001 & 53,2 & 0,003 \\
\hline Medline & 71,3 & 0,003 & 49,6 & 0,002 & 53,0 & $<0,001$ & 47,0 & 0,002 \\
\hline Ind.Farma. & 64,4 & 0,70 & 40,0 & 0,39 & 45,0 & 0,39 & 36,9 & 0,25 \\
\hline Opinião expert & 64,3 & 0,90 & 48,0 & 0,04 & 48,0 & 0,23 & 38,8 & 0,89 \\
\hline \multirow[t]{3}{*}{ Atividade docente } & 66,7 & 0,63 & 57,6 & 0,03 & 57,6 & 0,10 & 51,5 & 0,90 \\
\hline & $\begin{array}{l}\text { Conceito de teste } \\
\text { falso-positivo }\end{array}$ & $\mathrm{p}^{*}$ & $\begin{array}{l}\text { Conceito de } \\
\text { sensibilidade } \\
\text { diagnóstica }\end{array}$ & $\mathrm{p}^{*}$ & $\begin{array}{l}\text { Níveis de } \\
\text { evidência }\end{array}$ & $\mathrm{p}^{*}$ & $\begin{array}{l}\text { Interpretação } \\
\text { de gráfico de } \\
\text { metanálise }\end{array}$ & $\mathrm{p}^{*}$ \\
\hline & $\%$ & & $\%$ & & $\%$ & & $\%$ & \\
\hline \multicolumn{9}{|l|}{ Tempo de formação } \\
\hline Até 10 anos & 90,2 & 0,11 & 68,9 & 0,06 & 29,5 & 0,61 & 9,8 & 0,10 \\
\hline$>10$ anos & 81,1 & & 54,8 & & 26,0 & & 3,9 & \\
\hline \multicolumn{9}{|l|}{ Meio de atualização } \\
\hline Cochrane & 88,7 & 0,42 & 71,0 & 0,03 & 19,4 & 0,07 & 8,1 & 0,45 \\
\hline Medline & 87,0 & 0,33 & 67,5 & 0,009 & 25,2 & 0,30 & 6,1 & 0,98 \\
\hline Ind.Farma. & 87,5 & 0,03 & 58,8 & 0,20 & 27,5 & 0,81 & 5,0 & 0,08 \\
\hline Opinião Expert & 86,7 & 0,62 & 60,8 & 0,88 & 28,6 & 0,79 & 8,2 & 0,20 \\
\hline Atividade docente & 90,9 & 0.35 & 66,7 & 0,51 & 24,2 & 0,61 & 12,1 & 0,07 \\
\hline
\end{tabular}

*p significativo $<0.05$

de seleção de um artigo para avaliar tratamento (37/188 $59,7 \%$, p 0,001), associações entre a pergunta clínica e o tipo de estudo (33/188 - 53,2\%, p 0,003), e interpretação de teste diagnóstico (44/188 - mostrado na Tabela 3).

\section{DISCUSSÃO}

As informações obtidas mostram que as principais e mais frequentes fontes de educação permanente dos psiquiatras participantes consistem em congressos e periódicos científicos nacionais.

O Congresso Brasileiro de Psiquiatria é realizado anualmente e um de seus principais objetivos é promover a atualização dos especialistas na área. No entanto, um evento desta natureza, por mais que busque veicular informação científica de qualidade, está sujeito a uma gama de variáveis que dificultam este rigor. Desta forma, podemos inferir que a avaliação crítica da informação obtida nestas circunstâncias também pode estar sujeita às mesmas dificuldades, havendo necessidade de julgamento impetuoso das informações recebidas.

Quanto aos periódicos, tanto nacionais quanto internacionais, é necessário que se possa ter um julgamento criterioso, visto que a velocidade na produção de informações é muito grande, mas a qualidade das mesmas não é proporcionalmente correspondente. Assim, não é suficiente fazer uso dos periódicos apenas. É necessário dominar princípios de avaliação de qualidade dos artigos publicados.

O maior número de acertos entre os profissionais com até dez anos de formação para 50\% das questões está de acordo com um estudo realizado com 230 médicos ginecologistas/ obstetras que participaram voluntariamente durante um congresso brasileiro da área de Ginecologia e Obstetrícia em novembro de $2001^{9}$. O objetivo desse estudo foi avaliar o desempenho dos participantes nos testes sobre obstetrícia baseada em evidências, e os resultados mostraram que o escore médio 
de acerto dos recém-graduados (até cinco anos) era o mais alto quando comparado aos que tinham maior tempo de formação $(52,2 \pm 18,5$ vs. $42,9 \pm 17,1 ;$ p 0,07$)$. Nosso estudo utilizou método estatístico diferente, porém igualmente adequado à análise dos dados.

Os resultados encontrados sugerem que os médicos com menor tempo de graduação estão mais em sintonia com a tendência de crescimento no Brasil da utilização das ferramentas relacionadas à prática de ações em saúde baseadas em evidências; ao mesmo tempo, o baixo índice de acertos nas questões sobre níveis de evidência e metanálise entre estes mesmos profissionais chama a atenção e faz pensar que, apesar da maior veiculação destes conceitos, o conhecimento para o exercício desta prática ainda está aquém do necessário. O baixo índice de acertos destas duas questões aparece também entre os psiquiatras com atividade docente, e nenhuma das formas de atualização se mostrou positivamente associada a este conhecimento.

A escassa literatura passível de comparação com os resultados do presente estudo indica a necessidade de mais pesquisas com o intuito de verificar a capacitação dos psiquiatras para a tomada de decisão com base nas melhores evidências disponíveis. Esta capacitação faz com que o médico seja mais seletivo com relação a dados apresentados, principalmente por fontes de interesse (indústria farmacêutica, por exemplo), sendo diferenciado ao saber criticar uma evidência ${ }^{8}$.

O planejamento de educação continuada que proporcione aos psiquiatras os conhecimentos suficientes para a prática baseada em evidências significa levar benefício aos pacientes e proporcionar e manter a saúde, de acordo com os princípios éticos do exercício da medicina.

\section{REFERÊNCIAS}

1. Conselho Regional de Medicina do Estado de São Paulo. Código de ética médica: artigos sobre ética, direitos e deveres dos médicos e pacientes. São Paulo:CRM-SP; 2001.

2. Avezum A. Cardiologia baseada em evidências e avaliação crítica da literatura cardiológica: princípios de epidemiologia clínica aplicados à cardiologia. Rev Soc Cardiol Estado de São Paulo. 1996;3:241-59.
3. Evidence Based Medicine Working Group. Evidence based medicine: a new approach to teaching the practice of medicine. JAMA.1992;268(17):2420-5.

4. Drummond JP, Silva E. Medicina baseada em evidências. 3ạed. São Paulo: Atheneu; 1998.

5. Avezum A, Cavalcanti AB, Farsky P, Knobel M. Transferindo as evidências da pesquisa clínica para a prática cardiológica. Rev Assoc Med Bras. 2001;47(2):165-8.

6. Lim RF, Hsuing BC, Hales DI. Lifelong learning skills and online resources. Acad Psychiatry. 2006;30(6):540-7.

7. Glaziou P, Burls A, Gilbert R. Evidence based medicine and the medical curriculum. BMJ. 2008;337:a1253. [Editorials].

8. Sackett DL, Richardson WS, Rosenberg W, Haynes RB. Evidence-based Medicine - How to Practice and Teach EBM. London: Churchill Livingstone; 1997.

9. Sass N, Torloni MR, Soares BGO, Atallah AN. Continuing medical education in Brazil: What about obstetricians and gynecologists? Med Journal. 2005;123(1):5-10.

\section{CONTRIBUIÇÃO DOS AUTORES}

Isabela Yuri Tsuji, Liliana Cavalcanti Zamperin, participaram na concepção e delineamento deste estudo, da análise e interpretação dos dados assim como da redação deste texto, construção dos recursos gráficos. Selma Rumiko Tsuji e Valéria Garcia Caputo, participaram como orientadoras da autora principal na concepção e delineamento deste estudo, da análise e interpretação dos dados assim como da redação deste texto, construção dos recursos gráficos.

\section{CONFLITO DE INTERESSES}

Declarou não haver

\section{ENDEREÇO PARA CORRESPONDÊNCIA}

Isabela Yuri Tsuji

Rua Francisco Jose Capeline, 195

Jardim Aeroporto - Marília

CEP 17514-170 - SP

E-mail: isabelatsuji758@hotmail.com 